\title{
Real Time Tracking and Tracing of Shipment
}

\author{
Aakansha Vaidya ${ }^{1}$, Pooja Pitale ${ }^{2}$, Dipali Nikam ${ }^{3}$, Tanvi Goriwale ${ }^{4}$, Mr Ramdas Jare $^{5}$ \\ Student, Diploma in Computer Engineering, Pimpri Chinchwad Polytechnic, Pune, India ${ }^{1,2,3,4}$ \\ Lecturer, Diploma in Computer Engineering, Pimpri Chinchwad Polytechnic, Pune, India ${ }^{5}$
}

\begin{abstract}
The semi-trusted servers in cloud environment may outsource the files of their clients to some low expensive servers to increase their profit. To some extent, such behavior may violate the wishes of cloud users and impair their legitimate rights and interests. In this paper, a probabilistic challenge-response scheme is proposed to prove that the clients' files are available and stored in a specified cloud server. In order to resist the collusion of cloud servers, common cloud infrastructure with some reasonable limits, such as rational economic security model, semi-collusion security model and response time bound, are exploited. These limits guarantee that a malicious cloud server could not conduct a t-round communication in a finite time. So we proposed a system which will store data on cloud for specify time and then it will again restore automatically on the machine. The research reveals that the use of real time tracking technology in logistics is still at its infancy stage in UK, but with great potential to grow in the future[3]. We start analyzing motivations for cloud computing, providing also definitions and background for the following contributions. Then, we carefully analyze and discuss the properties of a monitoring system for the cloud. Cloud Computing has become an important aspect in today's world as technology has grown past all the boundaries and there is a need to connect resources and users without having physical connection. The high demand for data processing and leads to high computational requirement which is usually not available at the user's end.
\end{abstract}

Keywords: Pentium IV and Above, Lan, Hard Disk, Cloud Server, Compression, Decompression of a File, Compression Algorithms, Increasing Effective Data Density, Data Storage Space, Resource Usage or Transmission Capacity

\section{INTRODUCTION}

This paper focus on developing a system which will store data on cloud for specify time and then it will again restore automatically on the machine. This application will contain after the 1 GB data is deleted manually by the user. If there is no space on computer than message will go to user "unable to restore file because of insufficient storage". The files may be accessed in the cloud but all the files will remain encrypted till the USB device is plugged into the computer. This module helps to compress file and folder. We can send file over the internet and user decompress it.

\section{PROPOSED SYSTEM}

Let's consider a scenario where there is $500 \mathrm{MB}$ storage available in your system (computer) and you want to store 1 GB of File on that system. It will not be possible to store the file because of insufficient storage. So we proposed a system which will store data on cloud for specify time and then it will again restore automatically on the machine [2]. After the $1 \mathrm{~GB}$ data is deleted manually by the user. If there is no space on computer than message will go to user "unable to restore file because of insufficient storage". The files may be accessed in the cloud but all the files will remain encrypted till the USB device is plugged into the computer. The point of applying such method is to fully protect the files and avoid using one single password. The randomly generated passkeys are very complex combinations thus user will not be able to fully memorize them. The proposed system will detect the USB that contains the privatekey used for the files to be downloaded from the cloud. The connected devices may be PC, smart phones or tablets. Basically, any device that has a valid MAC address of integrated network adapter is included. The cloud computing is all about sharing of resources among users in real-time. Real-time refers to the sharing of data to be visible instantly to other users who has the authentication to see it.

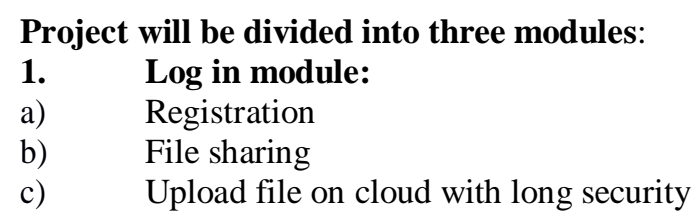

2. Compress file or folder module: This module helps to compress file and folder. We can send file over the internet and user decompress it. Compression algorithms reduce the redundancy in data representation thus increasing 
Vol. 8, Issue 1, January 2019

effective data density. Data compression is a very useful technique that helps in reducing the size of text data and storing the same amount of data in relatively fewer bits resulting in reducing the data storage space, resource usage or transmission capacity[4].

3. Decompress File or Folder module: This is reverse process of file. Easily get back original file with help of decompression. Decompression is the process of restoring compressed data to its original form. Data decompression is required in almost all cases of compressed data, including lossy and lossless compression. Similar to compression of data, decompression of data is also based on different algorithms.

\section{Following Steps are required for a project work in real phase:}

Step 1: Total Disk size $=80$ GB, Free Space $=500 \mathrm{MB}$

Step 2: Copying 1 GB File

Step 3: User will upload 500 MB file on Cloud. So now space is 1 GB

Step 4: User can now copy the $1 \mathrm{~GB}$ file

Step 5: Whenever user delete some file from disk again free space will created

Step 6: Uploaded file in step 3 will automatically download because of step 5

Step 7: Downloaded file automatically compresses (Reduce file size).

Step 8: Easily get back original file with help of decompression.

\section{LITERATURE SURVEY}

In the cloud computing environment, the Cloud Storage Providers (CSPs) offer paid storage space on its infrastructure to store customers' data. Since the CSPs are not necessarily trusted in cloud storage system, efficient and secure schemes should be built to constrain their malicious activities. For sensitive data, legitimate concerns are necessary when using cloud storage services. The failure of cloud storage server at Amazon results in the permanent loss of customer data. The tracking and tracing system is considered as industrial norms to provide customer services. The tracking system is usually considered as link between information systems and the physical realities [1].

\section{SCOPE OF THE PROJECT}

\section{Storage location security:}

In the multiple time storage re-outsourcing scenarios, the data owner will not be able to control the data re-outsourcing behavior of the malicious CSP and the location of its data is uncontrollable. Therefore, the clients' data may be stored in some servers controlled by its competitors or in some servers beyond the scope of legal protection. Then, some data security and privacy issues will arise.

\section{Low service quality:}

If the cloud storage service is provided in a multi-hop mode in storage re-outsourcing scenario, the CSPs may not be able to respond the request from their clients in time. Worse still, the CSPs will not be able to respond the clients when any CSP in the storage re-outsourcing chain is out of service. Also, the client's data will be stored in a lower payment data store which usually provides lower data security and quality of service guarantee.

\section{ARCHITECTURAL MODEL}

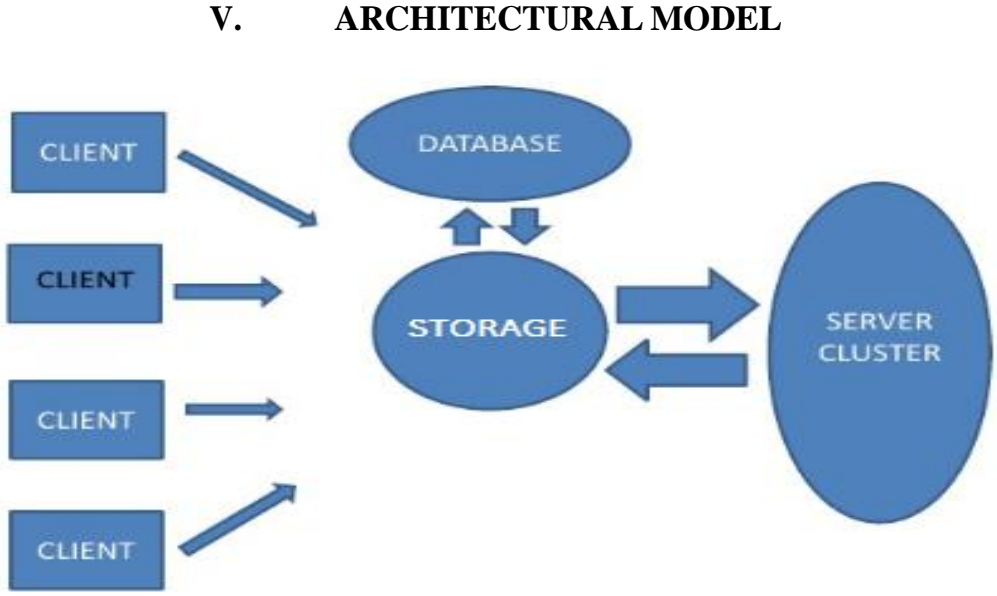

Fig 1. Database Server 
Vol. 8, Issue 1, January 2019

1. Server Cluster: Server cluster is used to stored data send by the storage for the particular time. Server cluster is the request provider to the client. Then after copying data into a clusters. Free space available into storage. Client will easily copy data into server storage.

2. Client: This is the primary Actor of model. First client send to request uploading file 1 GB of data. It is only send the request to server. They don't aware about any detail implementation.

3. Storage: In that multiple client send to request storage for storaging purpose then storage space is left only consider $500 \mathrm{mb}$.storage is requested to database retrieve particular data and update information regarding of free spaces.

4. Database: This is a main part of our project. Because all information stored into a a database. Whenever any data needed retrieving.

\title{
VI. TECHNICAL REQUIREMENT
}

\author{
A. Hardware Requirements: \\ 1. Hardware :Pentium IV and Above \\ 2. RAM :1GB \\ 3. Hard Disk :30GB (It Can Be Extended) \\ 4. LAN Cable $: 4$ \\ B. Software Requirements: \\ 1. Operating System: Windows and Linux \\ 2. Technology : Java and J2EE. \\ 3. Web Technologies :HTML, JSP, JavaScript, CSS, Servlet \\ 4. Web Server : Apache Tomcat \\ 5. Database : MySQL
}

\section{ADVANTAGES OF THEPROJECT}

1. Storage space is increased of device.

2. No manual restore is required it will automatically.

3. All cloud storage services review in this topic have desktop folder for Mac's and PC's and this allows a user to drag and drop files between the cloud storage and their local storage.

4. The storage file can be accessed from anywhere via internet connection.

5. Cloud storage costs about 3 cents per gigabyte to store data internally.

6. This application help to compress file and folder. We can send file over the internet and user decompress it

7. Agility: The cloud works in the distributed computing environment. It shares resources among users and works very fast.

8.High availability and reliability: Availability of servers is high and more reliable, because chances of infrastructure failure are minimal.

9. High Scalability: Means "on-demand" provisioning of resources on a large scale, without having engineers for peak loads.

10.Multi-Sharing: With the help of cloud computing, multiple users and applications can work more efficiently with cost reductions by sharing common infrastructure.

\section{LIMITATION}

1. Internet is required for cloud connectivity.

2. Doesn't work well in low speed connection.

3. The customer can only manage and control the application.

4. The data and services operated on top of that, not the backend infrastructure itself. 
Vol. 8, Issue 1, January 2019

\section{CONCLUSION}

Server side clients data re-outsourcing may cause some security problems in cloud storage environment. In this paper, the proposed probabilistic TIMER scheme will provide an efficient way to detect this malicious behavior of cloud servers. It adopts cryptographic assumptions and network delay to prevent servers from collusion in cloud storage, which will provide a strong incentive for the economically rational cloud server to store clients' data in their stores. We provide a security and performance analysis of our scheme.

\section{REFERENCES}

[1]. j.Gantz and d.reinsel, "the digital universe In 2020; Big data, bigger digitalshadows,and biggest growth in the far east,"http://www.emc.com/collateral/analyst-reports/idethe-digital-universe-in-2020.pdf,Dec 2012

[2]. M.O.Rabin,:'Fingerprinting by random polynomials,"Center for Research in Computing Technology,HarvardUniversity,Tech.Rep.Tech.Report TR-CSE-03-01,1981.

[3]. P. Barham, B. Dragovic, K. Fraser, S. Hand, T. Harris, A. Ho, R. Neugebauer, I. Pratt, A. Warfield, Xen and the art of virtualization, in: Proceedings of the 19th ACM Symposium on Operating Systems Principles, SOSP 2003, Bolton Landing, NY, USA, 2003, p. 177

[4]. M. Armbrust, A. Fox, R. Griffith, A.D. Joseph, R. Katz, A. Konwinski, G. Lee, D. Patterson, A. Rabkin, I. Stoica, M. Zaharia A view of cloud computing

[5]. J. Kaplan, W. Forrest, N. Kindler, Revolutionizing Data Center Energy Efficiency, McKinsey Company,Tech. Rep..

[6]. J.S. Chase, D.C. Anderson, P.N. Thakar, A.M. Vahdat, R.P. DoyleManaging energy and server resourcesin hosting centers Proceedings of the 18th ACM Symposium on Operating Systems Principles, ACM, New York, NY, USA (2001), pp. 103-116 